\title{
Ultraviolet Background Radiation from Beyond Pluto
}

\author{
Richard Conn Henry*t \\ Henry A. Rowland Department of Physics \& Astronomy, The Johns Hopkins University \\ E-mail: henryajhu.edu
}

The famous NASA mission New Horizons has now passed well beyond its historic mapping and scientific investigation of the planet Pluto, and is headed farther out into the Kuiper beltbeyond which, New Horizons will venture into the reaches of interstellar space! On its way there, New Horizons will carry out, on 2019 January 1, an investigation of Kuiper belt object (486958) $2014 \mathrm{MU}_{69}$. Once that solar-system object has been investigated, I am hoping to be able to use the ALICE ultraviolet spectrometer that is aboard New Horizons to carry out observations of the cosmic diffuse ultraviolet background radiation from the universe. ALICE is ideal for that purpose, first because it has wonderful $9 \AA$ spectral resolution, but, equally importantly, because of the ideal location of the New Horizons spacecraft that carries it, which will, by that time, be more than 43.4 Astronomical Units from the sun. That extreme distance from the sun is crucial for my purposes, because the sun is the source of a very bright general background of hydrogen Lyman $\alpha$ radiation that scatters off of the interstellar hydrogen and helium that is flowing through the solar system - and then — unfortunately - that solar Lyman $\alpha$ scatters to all wavelengths in the ALICE spectrometer! We need to go far enough from the sun so that that scattered Lyman $\alpha$ is small compared with the cosmic background, which is composed, to some degree, of the ultraviolet light from hot $\mathrm{O}$ and $\mathrm{B}$ type stars in our Galactic neighborhood scattering off interstellar dust in our part of the Galaxy-but a second component has a different origin, of unknown provenance: possibly from the radiative decay of the dark matter that forms the bulk of the mass of our Galaxy. Whatever its origin, if that second component of the ultraviolet background should extend below the Lyman limit of $912 \AA$, it would likely explain the reionization of the universe that followed shortly after the recombination that took place when the universe was only 379,000 years old. Giving us clues to that possibility would be an incredibly valuable byproduct of the wonderful New Horizons voyage to, and beyond, Pluto!

XII Multifrequency Behaviour of High Energy Cosmic Sources Workshop

12-17 June, 2017

Palermo, Italy

\footnotetext{
* Speaker.

${ }^{\dagger}$ Supported by Maryland Space Grant Consortium
} 


\section{Introduction}

The investigation of background radiation from the universe has a long history of producing major discoveries that have given us deep new understanding of the universe. In Figure 1 are shown the observations of that cosmic background radiation - at all wavelengths from the radio (where we see synchrotron radiation from high energy electrons in the magnetic field of our Galaxy) to the gamma ray (the integrated radiation from the quasars in the universe). The most prominent feature is the $3 \mathrm{~K}$ microwave background from the Big Bang explosion that began the universe 13.8 billion years ago. The discovery of that cosmic feature resulted in the award of Nobel prizes for physics-multiple Nobel prizes!

I have highlighted in red the narrow spectral region that is the focus of the present paper: the ultraviolet. This region is of critical importance for astrophysics, because electromagnetic radiation that is shorter in wavelength than $912 \AA$ is capable of ionizing hydrogen. One great current unsolved mystery of the universe is how the universe managed to get re-ionized after it had recombined following the big bang. This means that we are seeking a source of ionizing radiation, and the ultraviolet background radiation is the natural place to look for such photons.

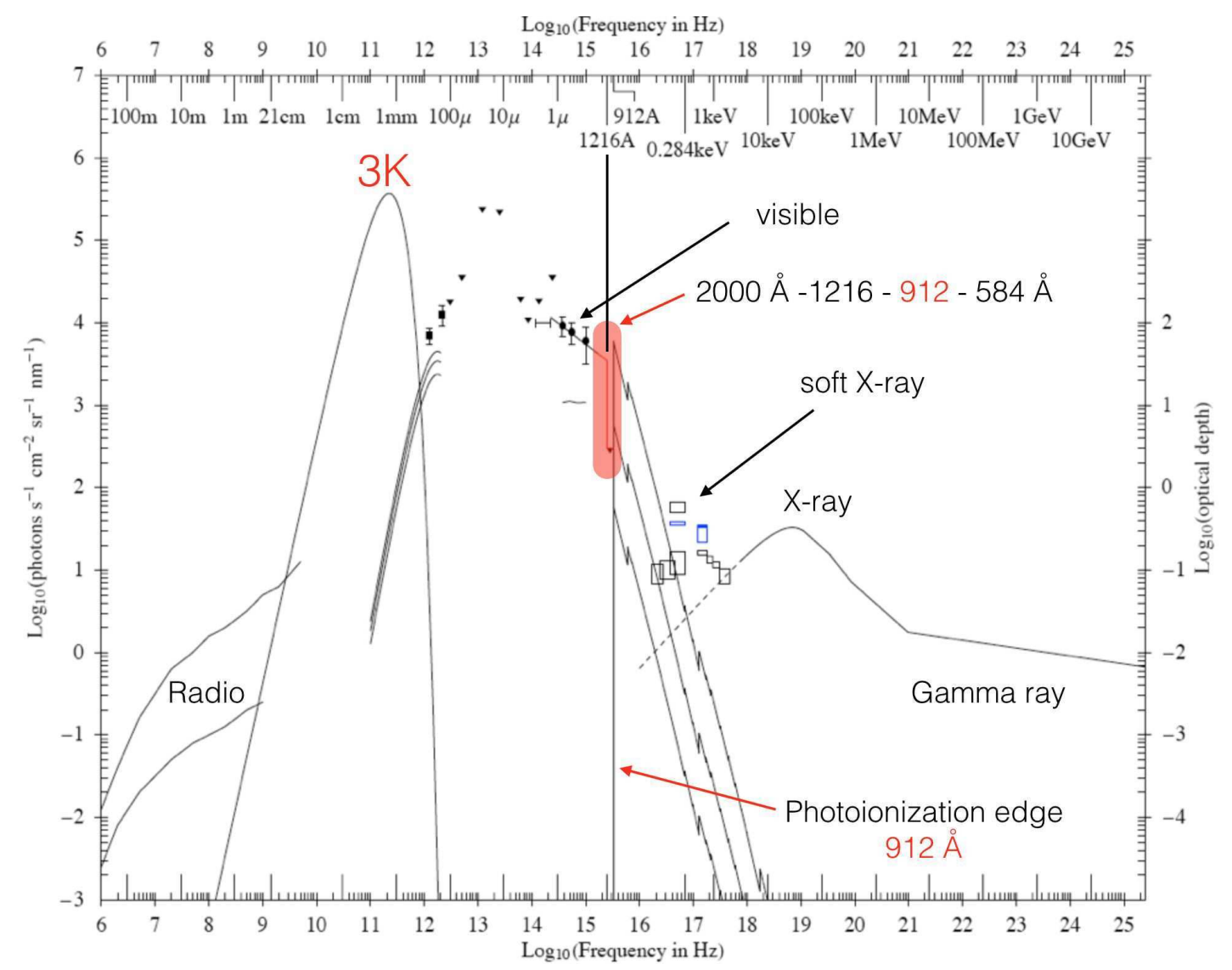

Figure 1: The spectrum of the cosmic background radiation, from the radio waves to the gamma rays. The three jagged lines give the logarithm of the optical depth of the interstellar medium (right figure legend) for three assumed column densities of the interstellar medium in the direction of observation. The ultraviolet spectral regions on which we focus in this paper are highlighted in red. 
Figure 1 is adapted from similar figures that I have presented at multifrequency meetings held in the past ([1]-[8]). In particular, I include the spectrum with a sharp drop right at Lyman $\alpha$, $1216 \AA$, that I once thought to be present in the ultraviolet background (Henry, R. C., 1999 ApJL 516, L49 [9]). Subsequent observations, above all with the ultraviolet spectrometers on the Voyager spacecraft (Murthy, J., Henry, R. C., Holberg, J. B., ApJSuppl 199:11, 2012 [10]), have eliminated that idea: we still do not know the answer!

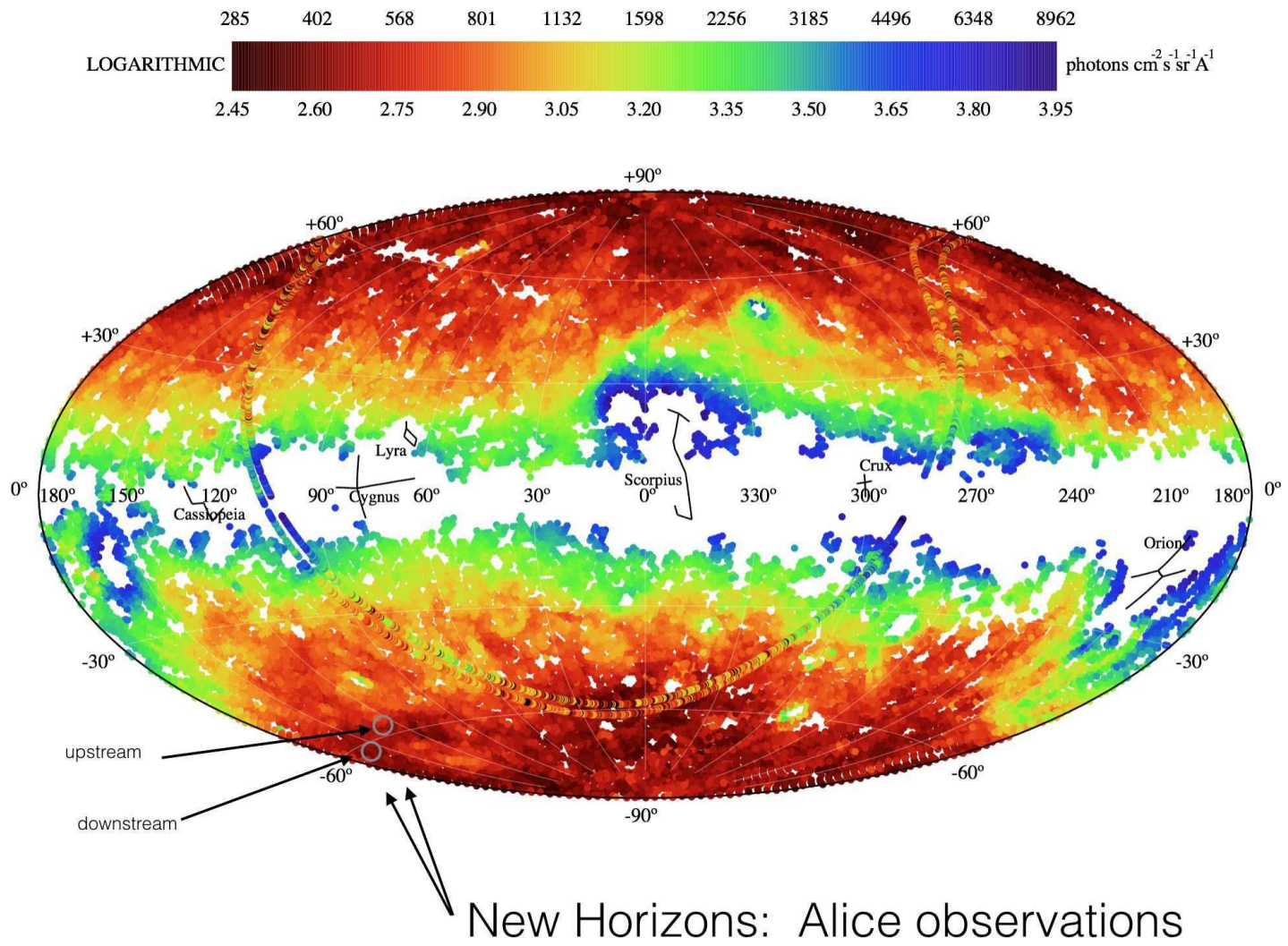

Figure 2: The locations of two recent observations (arbitrarily labelled "upstream" and "downstream") of the cosmic ultraviolet background using ALICE aboard New Horizons is shown against the similar observations over much of the sky using the FUV imager of the GALEX mission. Those GALEX observations are broad-band photometry (1300-1700 $)$; the crucial feature that ALICE provides is spectroscopy-and in particular, spectroscopy with a spectral resolution of $9 \AA$, which is sufficient to resolve the stellaratmosphere absorption lines that must appear if the cosmic diffuse ultraviolet background is dominated by the light of OB stars scattered off interstellar dust, as is often suggested to be the case. This figure is adapted from Figure 20 of Henry, Murthy, Overduin, and Tyler (2015) [11] where additional description and explanation can be found.

Of course some portion of the diffuse ultraviolet background is well understood to be simply starlight from the hot $\mathrm{O}$ and $\mathrm{B}$ type stars in our Galactic neighborhood scattering off of interstellar dust grains in our part of the Galaxy, but that there is a significant component having some different (and unknown) origin, was recently highlighted by Henry, Murthy, Overduin, and Tyler (2015) [11], who pointed to the possiblity that the second component is due to the decay of the 
dark matter particles in our galaxy with the emission of ultraviolet radiation, including radiation that is capable of ionizing the intergalactic gas. Observations of the diffuse UV background with ALICE aboard New Horizons could, I hope, settle the issue of the nature and origin of that second component.

\section{The Units to be used for spectra of the diffuse ultraviolet background radiation}

Diffuse background radiation can be presented using quite a variety of units, some of which are much better than others. The units that $I$ have used in Figure 1 are: $\log _{10}\left[\right.$ photons $\mathrm{cm}^{-2} \mathrm{~s}^{-1}$ $\left.\mathrm{sr}^{-1} \mathrm{~nm}^{-1}\right]$. The case for favoring these particular units was presented by Henry (1999 ApJL 52, L49 [9]), who drew attention to the pointed and cogent discussion of radiation units by Gehrels (1997, Nuovo Cimento, 112B,11 [12]). Henry, in his paper, was deliberately avoiding his beloved Ångtroms for the units of passband, but only in an attempt to be as "metric" as possible—but since then, I have joyfully reverted to "per $\AA$," in preference to "per nm," as the appropriate units for passband.

(I must note that the astrophysics community has unfortunately persisted in its widespread use of $v I_{v}$ for diffuse backgrounds, the defects of which units Gehrels meticulously pointed out in precise detail. But, that is another story.)

The units that are used for diffuse backgrounds by the ALICE team are Raleighs/nanometer $(\mathrm{R} / \mathrm{nm})$. The Raleigh is a unit of photon flux that was proposed in 1956 by Hunten, Roach, and Chamberlain [13]. One Rayleigh (the unit is symbolized by $R$, and is named after Robert John Strutt, $4^{\text {th }}$ Baron Rayleigh, 1875 - 1947) is $4 \pi \times 10^{-10}$ photons per square metre per second. Rayleighs are most useful if your instrument is immersed in a medium with equal amounts of radiation coming at you from all directions.

Let me display the units that define one Rayleigh per steradian, which is what we need, since the radiation field in which we are interested varies in brightness with direction of look:

$$
1 R=\frac{10^{6}}{4 \pi} \text { photons } \mathrm{cm}^{-2} \mathrm{~s}^{-1} \mathrm{sr}^{-1}=79,578 \text { photons } \mathrm{cm}^{-2} \mathrm{~s}^{-1} \mathrm{sr}^{-1}
$$

The Rayleigh was created by scientists focusing on isotropic line emission, but what we are concerned with in this paper is continuum emission, and so a further choice of units presents itself. I have already confessed to switching back to "per $\AA$ ” from the standard "per nm," and I prefer to stick with that choice: I am an astronomer, not a physicist, and besides, all atoms are about one $\AA$ in size!

So for continuum radiation, my preferred units are photons $\mathrm{cm}^{-2} \mathrm{~s}^{-1} \mathrm{sr}^{-1} \AA^{-1}$, which I have often in past papers referred to as "photon units."

So, to address our needs in the present paper, we have

$$
1 R / \mathrm{nm} \approx 80,000 \mathrm{ph} \mathrm{cm}^{-2} \mathrm{~s}^{-1} \mathrm{sr}^{-1} \frac{\mathrm{nm}^{-1}}{10 \AA \mathrm{nm}^{-1}}=8,000 \text { photons cm } \mathrm{cm}^{-2} \mathrm{~s}^{-1} \mathrm{sr}^{-1} \AA^{-1} .
$$

Over the decades, Henry has repeatedly focused on the fact that the cosmic ultraviolet background from the universe never has been convincingly measured to be, anywhere on the sky, less bright than about 300 photons $\mathrm{cm}^{-2} \mathrm{~s}^{-1} \mathrm{sr}^{-1} \AA^{-1}$, which corresponds to $0.0038 \mathrm{R} / \mathrm{nm}$. 


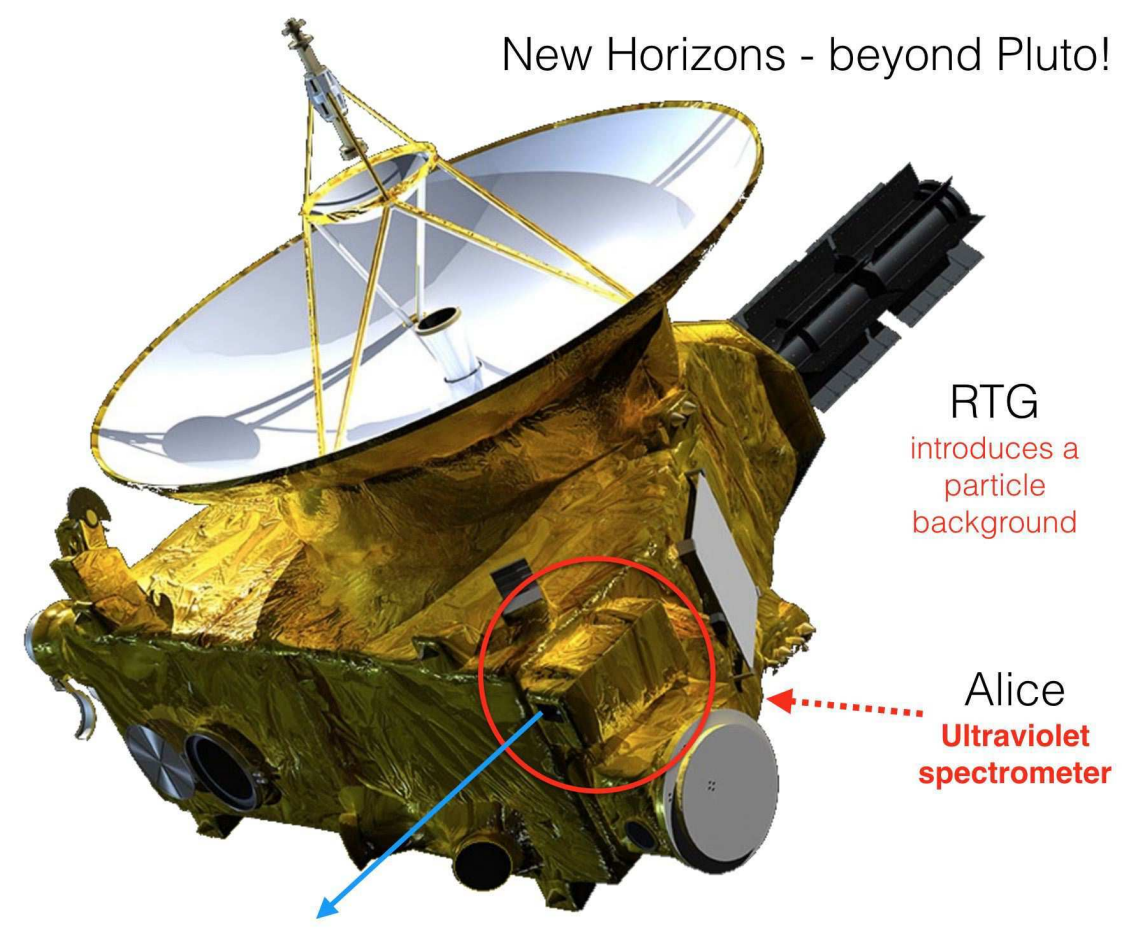

Figure 3: The ALICE ultraviolet spectrometer suffers from a strong particle background that is caused by its own radiothermal generator (RTG). The good news is that that particle background is due to radioactivity and so it is, statistically, perfectly pure noise-noise that can therefore be overcome simply by sufficiently long integrations. We expect that in order to detect, with adequate signal-to-noise, the faint background of ultraviolet radiation from the cosmos, observations of 24 hours or perhaps more will be necessary.

\section{GALEX observations of the diffuse ultraviolet background}

The analysis of the cosmic far-ultraviolet background (1300 -1700 $\AA$ ) by Henry et al. (2015) [11] is centered on the observations of the ultraviolet background that were made by the GALEX mission (Martin, Fanson, Schiminovich 2005) [14]. Those same observations have been analysed by Hamden et al. (2013) [15], who, like us, point to a second component to the background that is not easily attributable to starlight scattering from dust. The GALEX FUV sky is shown in Figure 2, which is adapted from Figure 20 of Henry, Murthy, Overduin, Tyler 2015 [11].

\section{Observing cosmic diffuse background with the ultraviolet spectrometer ALICE}

Figure 3 shows the location on the New Horizons spacecraft of the ultraviolet spectrometer that is called ALICE. The large dish antenna is for communication with the distant (and as of 2017, very distant!) planet Earth. Observations of the sky background have been made periodically over the years using the ALICE spectrometer. Figure 4 shows three such integrated spectra, taken over a span of some years. The three spectra were obtained during "Annual CheckOuts" ACO-1, ACO-2, and ACO-4 (no ALICE observations were made during ACO-3). That the spectra were dominated by grating-scattered Lyman $\alpha$ is demonstrated by the decline in brightness of 


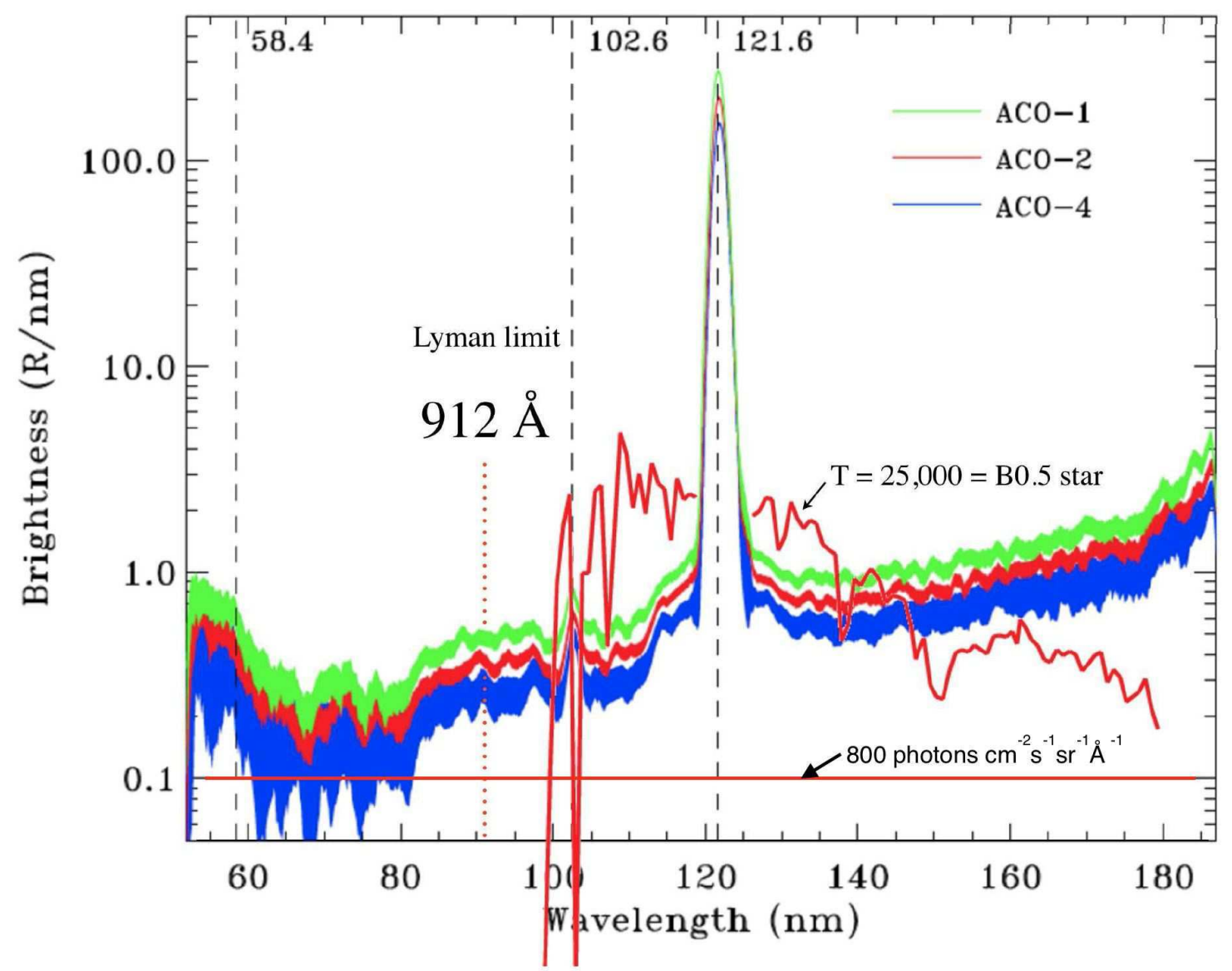

Figure 4: The three published New Horizons ALICE UV spectra, showing the decline in brightness as $\mathrm{NH}$ moves farther out in the solar system. Also appearing is the spectrum of a hot star, with a vertical scale exaggerated by a factor 1.5 to bring out more clearly the stellar spectral features that we hope to detect (or, more dramatically, fail to detect) in ALICE observations of the cosmic background. The vertical scale is in Rayleighs per nanometer. A brightness of $0.1 \mathrm{R} / \mathrm{nm}$ corresponds to $0.01 \mathrm{R} / \AA$ or 800 "photon units" (by which I mean photons $\mathrm{cm}^{-2} \mathrm{~s}^{-1} \mathrm{sr}^{-1} \AA^{-1}$ ). These spectra are dominated by the light of solar Lyman $\alpha$ scattering off of the interstellar hydrogen that is constantly flowing through the solar system. As New Horizons gets farther and farther from the Sun, this "noise" constantly diminishes, as is apparent in this figure, as all three spectra are of the same area of the sky, but from observations made many months apart (see Figure 5).

the radiation over that span of years, as New Horizons moved farther and farther from the source of the radiation-which is our own Sun. In Figure 5, I have plotted the brightness of the sky background at $912 \AA$, extracted from the spectra-measured over the years-that were presented in Figure 4, so that we can attempt to extrapolate to find out to what degree we can hope to improve matters as more years pass by: and New Horizons gets farther and farther from the sun: and as the scattered Lyman $\alpha$ decreases, and decreases, and decreases, in intensity.

All of the ALICE observations of blank sky that have so far been made are dominated by grating-scattered Lyman $\alpha$ radiation. Figure 4 shows the three spectra that are currently publicly available. They show no resemblance to the spectrum of a B 0.5 star that I have included 


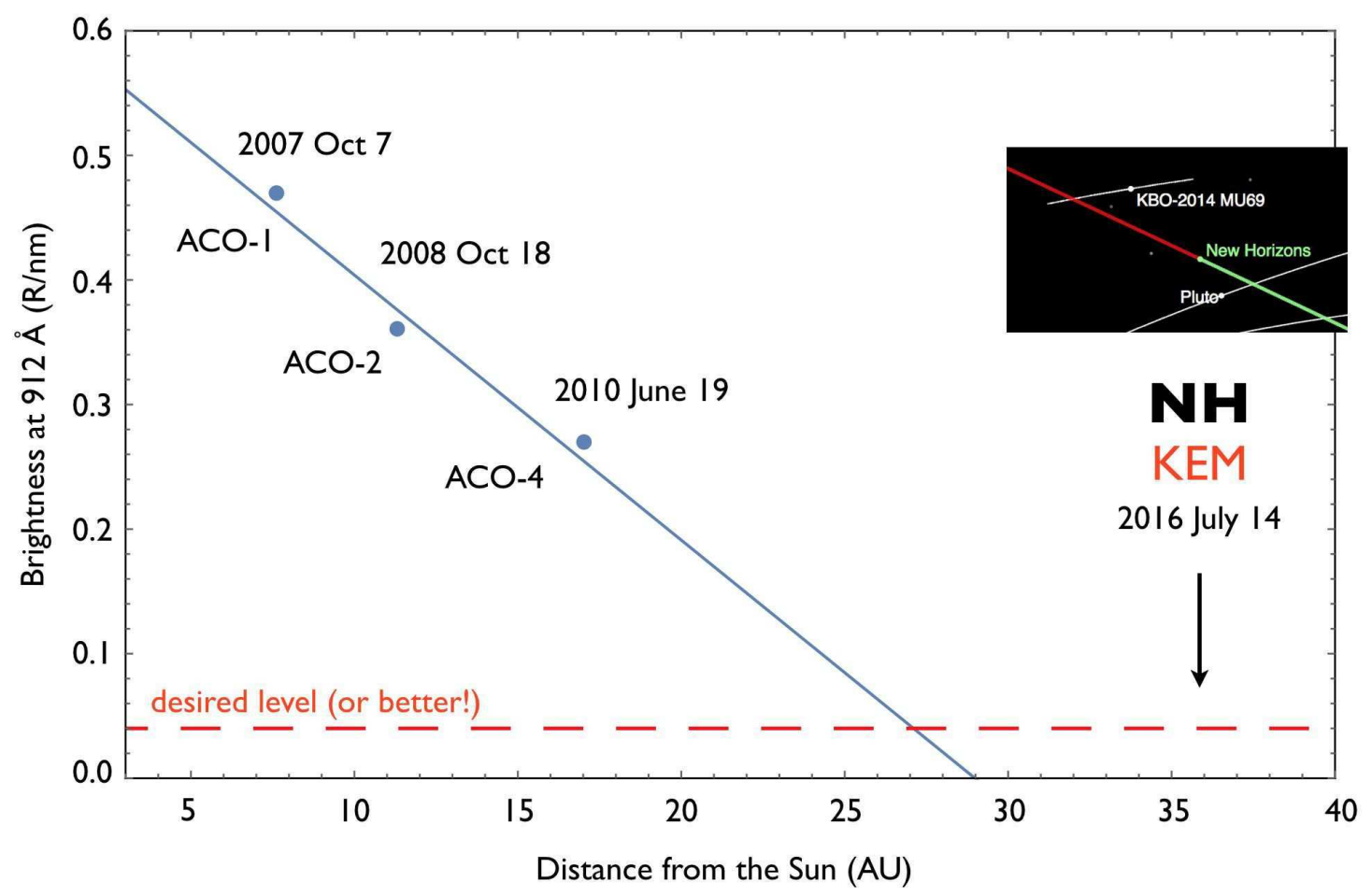

Figure 5: As the New Horizons spacecraft proceeded farther and farther from the Sun, the instruments that are on board were, normally, not taking measurements. But every so often, so as to verify their health, exercises took place, called "Annual CheckOuts" or ACO's. ACO-1, ACO-2, and ACO-4 included exercise of the ALICE ultraviolet spectrometer. During those ACO's, the spacecraft was rotated so that ALICE scanned the same great circle around the sky each time. The scan path was chosen to avoid bright ultraviolet-emitting stars. As we saw in Figure 4, the integrated spectra decreased in brightness from each ACO to the next, demonstrating that the signal at all wavelengths was dominated by non-astrophysical sources - in particular, by one non-astrophysical source, solar Lyman $\alpha$ (which is extremely bright) scattering from the interstellar hydrogen that is constantly flowing through our solar system, and then (unfortunately) scattering to other wavelengths from ALICE's grating. I have plotted in this figure the measured brightnesses near $912 \AA$ as a function of the distance of New Horizons from the Sun-which is the source of that nasty grating-scattered Lyman $\alpha$. The trend is suggested by a straight line, but what is really expected is a somewhat slower decline. As of 2017, unpublished ACO spectra show a decline to the encouraging level of about 800 photons $\mathrm{cm}^{-2} \mathrm{~s}^{-1} \mathrm{sr}^{-1} \AA^{-1}$ (or $0.1 \mathrm{R} / \mathrm{nm}$ units), and lower still at somewhat shorter wavelengths. Those spectra have not yet been released. They are noisy, as the exposures were only one hour in length. That is unimportant; the important thing is that the non-astrophysical background is still steadily decreasing! It will, necessarily, decrease more and more as New Horizons departs the solar system. 


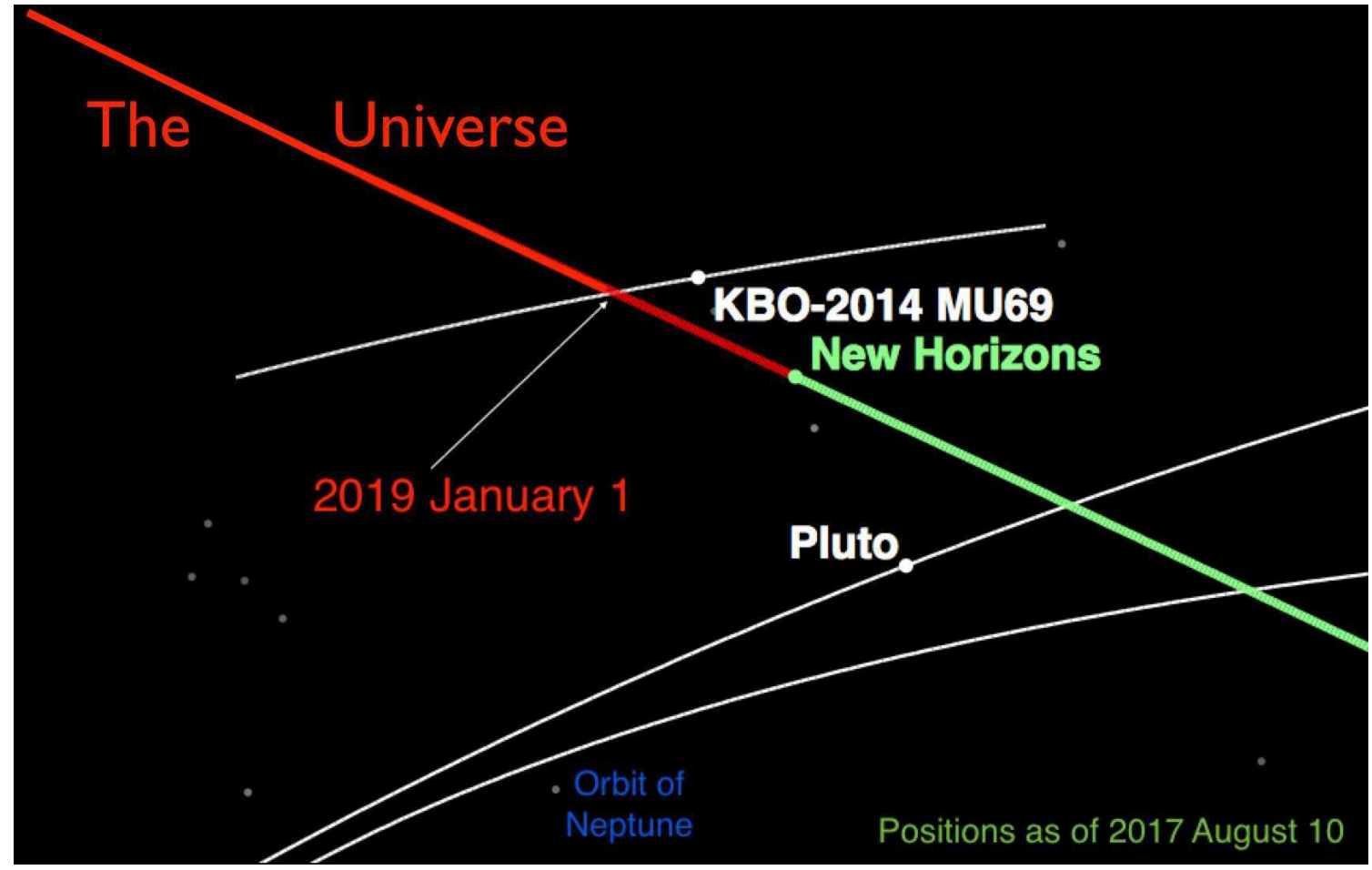

Figure 6: New Horizons is past Pluto now, and is headed for (486958) $2014 \mathrm{MU}_{69}$, after which we hope to observe the ultraviolet background with ALICE. The good news is that the noise (the solar gratingscattered Lyman $\alpha$ ) can only decrease with time, as New Horizons gets farther and farther from the Sun. Eventually the cosmic signal will equal and then exceed the noise. The noise has two components: the solar radiation, which should decrease continually as time passes and New Horizons is farther and farther from the Sun, and particle background from the RTG that provides power to the mission. The RTG background should be statistically extremely well-behaved, meaning that with a long enough integration it can be removed with arbitrary accuracy. Exposures of about a week may be enough to do the trick!

in the figure: so the radiation that is seen is not the cosmic background that I am searching for, but rather is simply grating-scattered solar system Lyman $\alpha$ radiation that has been scattered from the interstellar hydrogen that is constantly flowing through our solar system. But notice that the three ALICE spectra, which are all of the same great circle over the sky, are not all of the same brightness: the brightest, ACO-1 (green spectrum) was taken earliest in the mission, while the ACO-2 observation (red spectrum) was taken a year later, and finally, the ACO-4 spectrum (blue) was taken much later, by which time New Horizons was much farther from the sun-and so the scattered solar Lyman $\alpha$ radiation is much reduced. Our hope is that, in years to come, that reduction in scattered Lyman $\alpha$ background noise will continue to the point that a $9 \AA$ resolution spectrum of the true cosmic UV background can be obtained: we hope allowing diagnosis of its cosmic origin: the scattered light of galactic OB stars, plus-I hope and expect—something more!

\section{Conclusion}

The most important single problem in all of physics today is the determination of the nature of the mysterious dark matter that has been discovered to be present in our universe in vast 
amounts. Despite enormous efforts, its nature remains, at the present moment, the deepest of mysteries.

But it was just a few decades ago that we did not even know of the existence of that dark matter! For it was believed in my youth that the universe consisted of only spacetime and matter. By "matter" was meant that which we-these days—have come to refer to-now—as "baryonic matter," so as to distinguish that stuff of which we ourselves (as well as Pluto; and all the stars; and ...) are made, from the mysterious new dark matter (which is only known to exist because of its gravitational effects on the observed baryonic matter).

And by the way!

It is the newly-discovered dark matter that dominates: not the baryonic matter, which, such a short time ago, we thought was all that there is!

When one looks at a photograph of any galaxy-for example our neighbor, the huge Andromeda galaxy - it is rather as if you were looking at the ocean — but that all that you were able to see of the sea was the whitecaps. The Andromeda galaxy is a "whitecap" atop (or rather amidst) a vastly more massive blob of the dark matter; matter that is, still today, of utterly unknown composition! And conceivably, New Horizons could provide the first clue to its nature!

What a time to be alive and doing physics and astronomy!

Let me list (as of 2017), from the smallest component to the largest, our current inventory of the composition of $100 \%$ of what we refer to as the universe:

$0.5 \%$ : You; me; Pluto, our sun and all the stars; the interstellar gas clouds; and also all of those galaxies (such as Andromeda) that there are out there: and there's billions of them! And yes, that was half of one percent!

$4.4 \%$ : more matter (much more!) of exactly the same kind (that is, baryonic), but all (or almost all?) in the form of ionized intergalactic gas. This ionized gas is most of the baryonic matter in the universe - most of the baryonic matter in the universe was first detected by Meekins, Fritz, Chubb, Friedman, and Henry, "X-Rays from the Coma Cluster of Galaxies," Nature 231, 107 (1971) [16] by its X-ray emission from the Coma cluster of galaxies. Their discovery was confirmed by Gursky et al. (1971) [17] . That the radiation came from hot baryonic matter soon became apparent: "X-Radiation from Clusters of Galaxies: Spectral Evidence for a Hot Evolved Gas,” P. J. Serlemitsos, B. W. Smith, E. A. Boldt, S. S. Holt, and J. H. Swank, ApJ, 211:L63, $1977[18]$.

$26.8 \%$ : the dark matter-the characterization of which is (at least I hope that it is) the subject of the present paper!

And finally...

$68.3 \%$ : the now famous Dark Energy, $\Lambda$, which is (most likely) exactly what was predicted by Albert Einstein just over 100 years ago in his theory of General Relativity: Einstein asserted that $G_{\mu}{ }^{v}+\Lambda_{\mu}{ }^{v}=\frac{8 \pi}{c^{4}} T_{\mu}{ }^{v}$, where $\Lambda$ is a completely uniform mass density that, remarkably, does not change even as the universe expands and expands—so, it is emphatically not "particles!"

That all adds up to $100 \%$, and it seems that the universe is close to, or is right on, exactly that 
magic edge on one side of which the universe would be humungous (but not infinite) and on the other side of which the universe would, in fact, actually be infinite.

Which of these two it is, is deeply consequential from every point of view from physics to philosophy to religion. For if the universe is actually infinite, then there is an infinity of planets identical at this moment to our Earth, including "me" typing these lines on a Macintosh. And infinitely many more in which I now type $*(\%)$ just to show off, but also infinitely many more in which I do other things. In terms of the significance and meaning of your life and mine, what could be more consequential? And perhaps we will never know if the universe actually IS infinite. Moreover, even if the universe turns out to be detectably "closed" (finite) there could be other universes-whatever that may mean, if anything!

We have seen that the diffuse cosmic ultraviolet background radiation could conceivably hold important astrophysical information, and so surely deserves detailed study. How likely is it that the ultraviolet background originates (in part) in emission from the dark matter? Well, the mysterious component of the ultraviolet background radiation must come from something - and the dark matter seems to be the only available candidate source. I am optimistic that New Horizons could produce the greatest result in astrophysics of this century!

When, with my colleagues at the US Naval Research laboratory in 1970, I was planning targets for our next X-ray rocket flight, I told them that when I was a Toronto graduate student, my professor, Sidney van den Bergh, came back from a meeting in California and told us all that the Coma cluster of galaxies seemed to contain vast quantities of what was rather ridiculously called "missing matter." And that as a graduate student at Princeton I told that to George Field, and suggested a way to detect that matter if it was hot ionized gas. Field had a much better method of detection, and we worked on it together. So now the chance had come to see if it was there! Well, it was not! Yes, we detected X-rays [16] . Yes, the X-rays did turn out to be from ionized gas. But it was not nearly enough to bind the Coma cluster! I was disappointed. It missed me completely that although it was not enough to bind the Coma cluster, it was far more baryonic matter than there was in all the galaxies in that cluster!

Today, I don't want to miss discovering the nature of the dark matter, if there is any chance of doing so by measuring the spectrum of the cosmic ultraviolet background. Because of its unique location in the outer reaches of our solar system, New Horizons, with its ALICE ultraviolet spectrometer, is an intellectual resource of, at least potentially, incalculable value to the human race. Any failure to fully utilize this resource would be a combination of farce and tragedy.

\section{Acknowledgements}

I thank, with deepest sincerity, those who implemented so very well the marvellous ultraviolet spectrometer ALICE that is on board the New Horizons mission [19, 20] !

\section{References}

[1] Richard Conn Henry, "Progress in Understanding the Diffuse UV Cosmic Background," Mem. S.A.It., 83, 409, 2012 (arXiv: 1205.04430v1 [Astro-ph.GA] 2 May 2012)

[2] R. C. Henry, "Diffuse UV Background: GALEX Results," Memorie della Societa Astronomica Italian, eds. F. Giovannelli and L. Sabau-Graziati, 81, Nos. 1-2, 63, 2010 
[3] R. C. Henry, "Bright Light from Dark Matter (Baryonic and Non-Baryonic)," Chinese Journal of Astronomy and Astrophysics, 6, Suppl.1, 40-46 (Frascati Workshop 2005), 2006

[4] R. C. Henry, "Progress in Understanding the Diffuse UV Background," Chinese Journal of Astronomy and Astrophysics, 3, Supplement, 53, 2003

[5] R. C. Henry, "Non-Microwave Background After BOOMERanG," invited paper presented at "Multifrequency Behaviour of High Energy Cosmic Sources," Memorie della Societa Astronomica Italian, eds. F. Giovannelli and L. Sabau-Graziati, 73, No. 4, pp. 954-964, 2002

[6] R. C. Henry, "Cosmic Background Radiation," invited paper presented at "Multifrequency Behaviour of High Energy Cosmic Sources," Memorie della Societa Astronomica Italian, eds. F. Giovannelli and L. Sabau-Graziati, 73, No. 3, pp. 67-75, 2002

[7] R. C. Henry, "The Diffuse Ultraviolet Background Radiation," invited paper presented at "Multifrequency Behaviour of High Energy Cosmic Sources," Memorie della Societa Astronomica Italian, eds. F. Giovannelli and L. Sabau-Graziati, 70, No.s 3/4, pp. 825-830, 1999

[8] R. C. Henry, "Hopkins Ultraviolet Background Explorer," paper presented at "Multifrequency Behaviour of High Energy Cosmic Sources," Memorie della Societa Astronomica Italian, eds. F. Giovannelli and L. Sabau-Graziati, 70, No.s 3/4, pp. 1347-1352, 1999

[9] R. C. Henry, “Diffuse Background Radiation,” ApJL 516:L49-L52, 1999 May 10

[10] J. Murthy, R. C. Henry, J. B. Holberg, "The Voyager observations of the Diffuse Far Ultraviolet Radiation Field," ApJS, 199:11, 2012

[11] R. C. Henry, Jayant Murthy, James Overduin, Joshua Tyler, "The Mystery of the Cosmic Diffuse Ultraviolet Background,” ApJ 798:14 (25pp), 2015 January 1

[12] N. Gehrels, "Use of $v F_{v}$ spectral energy distributions for multiwavelength astronomy," Il Nuovo Cimento, 112B, N. 1, Gennaio 1997

[13] D. M. Hunten, F. E. Roach, J. W. Chamberlain, "A photometric unit for the airglow and aurora," Journal of Atmospheric and Terrestrial Physics, 8 (6): 345-346, 1956

[14] D. C. Martin, J. Fanson, D. Schiminovich et al., “The Galaxy Evolution Explorer,” ApJ 619:L1-L6, 2005 January 20

[15] E. T. Hamden, D. Schiminovich, M. Seibert, “The Diffuse Galactic Far-Ultraviolet Sky,” ApJ 779:180, 2013 December 20

[16] J. F. Meekins, G. Fritz, T. A. Chubb, H. Friedman, R. C. Henry, "X-Rays from the Coma Cluster of Galaxies," Nature 231, 107, 1971

[17] H. Gursky, E. Kellogg, S. Murray, C. Leong, H. Tannenbaum, R. Giacconi, “A Strong X-Ray Source in the Coma Cluster Observed by UHURU,” ApJ 167:L81,1971 August 1

[18] P. J. Serlemitsos, B. W. Smith, E. A. Boldt, S. S. Holt, J. H. Swank, "X-Radiation from Clusters of Galaxies: Spectral Evidence for a Hot Evolved Gas,” ApJ, 211:L63, 1977

[19] S. Alan Stern, D. C. Slater, J. Scherrer, J. Stone, G. Dirks, M. Versteeg, M. Davis, G. Randall Gladstone, Joel W. Parker, L. A. Young, and O. H. W. Siegmund, "ALICE: The Ultraviolet Imaging Spectrograph Aboard the New Horizons Pluto-Kuiper Belt Mission," Space Science Reviews (2008) 140: $155-187$

[20] G. Randall Gladstone, S. Alan Stern, Wayne R. Pryor, "New Horizons Cruise Observations of Lyman- $\alpha$ Emissions from the Interplanetary Medium,” doi 10.1007/978-1-4614-6384-9_6 